\title{
Deformed Clifford algebra and supersymmetric quantum mechanics on a phase space with applications in quantum optics
}

\author{
I. Buğdayc** and A. Verçint \\ Department of Physics, Ankara University, \\ Faculty of Sciences, 06100, Tandoğan-Ankara, Turkey
}

(Dated: November 12, 2018)

\begin{abstract}
In order to realize supersymmetric quantum mechanics methods on a four dimensional classical phase space, the complexified Clifford algebra of this space is extended by deforming it with the Moyal star-product in composing the components of Clifford forms. Two isospectral matrix Hamiltonians having a common bosonic part but different fermionic parts depending on four real-valued phase space functions are obtained. The Hamiltonians are doubly intertwined via matrix-valued functions which are divisors of zero in the resulting Moyal-Clifford algebra. Two illustrative examples corresponding to Jaynes-Cummings-type models of quantum optics are presented as special cases of the method. Their spectra, eigenspinors and Wigner functions as well as their constants of motion are also obtained within the autonomous framework of deformation quantization.
\end{abstract}

Keywords: Clifford algebra, supersymmetric quantum mechanics, deformation quantization, Wigner functions, Jaynes-Cummings models

PACS numbers: 45.20.Jj, 11.30.Pb, 03.65.Fd

*Electronic address: bugdayci@science.ankara.edu.tr

${ }^{\dagger}$ Electronic address: vercin@science.ankara.edu.tr 


\section{INTRODUCTION}

Moyal star-product formalism, more generally deformation quantization, provides useful tools for describing the phase space formulation of quantum mechanics [1, 2]. Spin and relativistic quantum mechanics can also be considered in this framework by making use of an additional fermionic star product [3]. It is seen that a combination of the Clifford product as fermionic part and Moyal star-product as bosonic part plays an important role in these regards. This approach can also be adopted to realize the methods of supersymmetric quantum mechanics [4, 5, 6, 7] on a phase space. Henselder has already shown how such an approach can be accomplished on a two-dimensional $(2 D)$ phase space in a recent study [8]. The main goal of the present work is to show that such a programme can be realized on a $4 D$ phase space in its full generality and rich applicability potential.

In this paper, we first study the deformation of the complex Clifford algebra defined on a $4 D$ phase space of a classical Hamiltonian system. The deformation is performed in composing the components of Clifford forms with the Moyal star-product. This enables us to obtain two iso-spectral matrix Hamiltonians, each being a sum of factorized terms. The Hamiltonians depend on four real-valued phase-space functions and they are general enough for modeling various systems. Moreover, the Hamiltonians are also doubly intertwined in such a way that the intertwining matrix-valued phase-space functions are divisors of zero in the resulting deformed algebra. By composing the intertwining matrices with their conjugates, we obtain a constant of motion for each system. Well-known Jaynes-Cummingstype models of quantum optics [9, 10, 11], which are the main subject of recent experiments

to understand the quantum nature of atom-field interactions [12], emerge as special cases of our method. These are studied in detail as the illustrative examples. Their phase-space characteristics such as their spectra, eigenspinors and related Wigner functions as well as their classical limits and constants of motion are obtained in an autonomous way, without any reference to the standard tools of quantum mechanics.

The paper is structured into two main parts. The first part consists of the following three sections where our method is fully developed. In Sec. II the Clifford algebra structure of $4 D$ classical phase space and its complexification are studied and the basics of Moyal star-product are briefly reviewed. The deformation of the algebra and its application for establishment of supersymmetry techniques on the phase space are taken up in Sec. III 
and its physical consequences are studied in Sec. IV. Sec. V, which constitutes the second part of the study, is entirely devoted to the applications of the method in quantum optics. Concluding remarks are given in the final section.

\section{CLIFFORD ALGEBRA ON A PHASE-SPACE}

Any vector space on which a non-degenerate inner product is defined has a Clifford algebra structure. Tangent (or cotangent) space of any (pseudo) Riemannian manifold is the best place where efficiency of Clifford calculus can be observed [13]. In this regard the symplectic manifold that we shall consider in this and the following section can be any even-dimensional manifold. However, in order to be able to do quantization consistently in such a framework, one needs the star product of deformation quantization (see subsection IIC) which requires globally defined coordinates. For this reason and for physically relevant applications, we shall confine the investigation to $4 D$ flat cases. Our method can easily be extended to an arbitrary flat symplectic manifold. For definiteness, we take our phase space to be a $4 D$ symplectic manifold $\mathcal{M}$ topologically equivalent to $\mathbb{R}^{4}$ and denote the linear space of complex-valued, smooth (differentiable to all orders) functions defined on it by $\mathcal{F}$. Real-valued elements of $\mathcal{F}$ are the classical observables of Hamiltonian mechanics.

At each point of $\mathcal{M}$ two $4 D$ vector spaces, the tangent space and (its dual) the cotangent space are naturally defined. Elements of the latter are 1-forms acting linearly on the elements of the former. $\mathcal{M}$ carries two distinguished structures defined by two non-degenerate, second rank tensor fields: the symplectic 2-form $\Omega$ (which is also closed) and the Euclidean symmetric metric tensor $g$. They are non-degenerate in the sense that each defines a vector space isomorphism between the tangent and cotangent spaces. The elements associated by these isomorphisms are called symplectic and metric dual of each other. The metric dual of a vector field $x$ is a 1 -form $\tilde{x}$ defined, for a given vector field $y$, as $\tilde{x}(y)=i_{y}(\tilde{x})=g(x, y)$. Here $i_{y}$ denotes the so-called interior derivation (or interior product) whose action on an arbitrary $k$-form (a totally anti-symmetric covariant tensor) $\beta$ is defined, for arbitrary vector fields $x_{1}, \ldots x_{k-1}$, by $\left(i_{y} \beta\right)\left(x_{1}, \ldots, x_{k-1}\right)=k \beta\left(y, x_{1}, \ldots x_{k-1}\right) . \Omega$ and $g$ at each point of a given neighborhood endow the tangent space with structures of a symplectic vector space and an inner product space, respectively. According to the Darboux theorem [14], in each neighborhood of $\mathcal{M}$ one can define the canonical coordinates $(\mathbf{q}, \mathbf{p})=\left(q_{1}, q_{2}, p_{1}, p_{2}\right)$ which 
lead to $\Omega=\sum_{j} d q_{j} \wedge d p_{j}$. Here $\wedge$ denotes the bilinear and associative exterior product which satisfies

$$
\alpha \wedge \beta=(-1)^{j k} \beta \wedge \alpha,
$$

for arbitrary $j$-form $\alpha$ and $k$-form $\beta$. $\Omega$ eventually leads to the Poisson bracket (PB)

$$
[F, G]_{P}=\sum_{k=1}^{2}\left(\partial_{q_{k}} F \partial_{p_{k}} G-\partial_{p_{k}} F \partial_{q_{k}} G\right)
$$

of Hamiltonian mechanics. We henceforth use the abbreviation $\partial_{q_{i}} \equiv \partial / \partial q_{i}$ and denote the generic elements of $\mathcal{F}$ by capital Latin letters. On the right-hand side of (1), the following ordinary commutative and associative pointwise product of functions is essential:

$$
\left(F_{1} F_{2}\right)(\mathbf{q}, \mathbf{p})=\left(F_{2} F_{1}\right)(\mathbf{q}, \mathbf{p})=F_{1}(\mathbf{q}, \mathbf{p}) F_{2}(\mathbf{q}, \mathbf{p})=F_{2}(\mathbf{q}, \mathbf{p}) F_{1}(\mathbf{q}, \mathbf{p}) .
$$

\section{A. Clifford Bundle and Complexification}

The linear space of all forms constitutes the exterior algebra with respect to exterior product, which (like the tensor algebra) is a $\mathbb{Z}$-graded associative algebra. Although, unlike the tensor algebra, the exterior algebra is finite dimensional $\left(2^{n} D\right.$ when the dimension of $\mathcal{M}$ is $n$ ) its $\mathbb{Z}$-gradation is inherited from the tensor algebra. In particular, any $k$-form for $k>n$ is zero and the zero element of the exterior algebra is the only homogeneous element of every degree greater than $n$ (see also [13] pp. 7-8).

The Clifford product $*_{C}$, defined for a 1-form $\tilde{x}$ and an arbitrary form $\beta$ by

$$
\tilde{x} *_{C} \beta=\tilde{x} \wedge \beta+i_{x} \beta,
$$

turns the exterior algebra into a $\mathbb{Z}_{2}$-graded associative algebra called the Clifford algebra (also known as the geometric algebra [15, 16]. See also [17] for applications in Hamiltonian mechanics). By associativity, the rule (2) suffices to completely determine $*_{C}$ on arbitrary forms. $\mathbb{Z}_{2}$-gradation means that the whole algebra is, as a linear space, a direct sum of the spaces of the odd and even forms such that the latter has a subalgebra structure. The Clifford product of a $j$-form $\alpha$ and $k$-form $\beta$ is, in general, an inhomogeneous form consisting of a sum of $\ell$-forms such that $\ell=j+k, j+k-2, \ldots,|j-k|$. The exterior bundle (union of exterior algebras) equipped with the $*_{C}$-product in the fibres is called the Clifford bundle. The Clifford commutator, defined by

$$
[\alpha, \beta]_{C}=\alpha *_{C} \beta-\beta *_{C} \alpha,
$$


is bilinear, antisymmetric and satisfies the Jacobi identity. Each fibre of the Clifford bundle acquires a Lie algebra structure with this bracket. In the context of Clifford algebra, we shall mainly adopt the conventions of [13], except for the representation of the Clifford product. More conventional versions of this product are $\tilde{x} *_{C} \beta=\tilde{x} \vee \beta$ and juxtaposing the factors.

Tangent spaces can be complexified by replacing the field of real numbers $\mathbb{R}$ by the field of complex numbers $\mathbb{C}$. Then $g$ can be extended by $\mathbb{C}$-linearity to the complex-valued, symmetric and non-degenerate (guaranteed by the non-degeneracy of $g$ ) bilinear map $g^{\mathbb{C}}$. As $\mathbb{C}$ is algebraically closed, $g^{\mathbb{C}}$ is not characterized by any signature (even if $g$ had any non-Euclidean signature) and hence the structure of complex algebra depends only on the dimension. In our case, we consider $2^{4} D$ real Clifford algebra $\mathbf{C}_{4,0}(\mathbb{R})$ which is isomorphic to the algebra of $2 \times 2$ quaternion matrices ([13], p.80). But from here on, we shall deal with its complexification $\mathbf{C}_{4}(\mathbb{C})$ which is known to be isomorphic to the algebra of $4 \times 4$ complex matrices.

\section{B. The Primary SUSY Structure}

The $2^{4} \mathrm{D}$ complex algebra $\mathbf{C}_{4}(\mathbb{C})$ is generated by 1 and by the complex orthonormal basis $\left\{e^{1}, e^{2}, e^{3}, e^{4}\right\}$ such that

$$
e^{j} *_{C} e^{k}+e^{k} *_{C} e^{j}=2 \delta^{j k}
$$

where the Kronecker symbols denotes the components of the (inverse) metric $g^{\mathbb{C}}\left(e^{j}, e^{k}\right)=\delta^{j k}$. We start out our analysis by the Clifford 1-form

$$
\omega=W_{1} e^{1}+W_{2} e^{2}+P_{1} e^{3}+P_{2} e^{4}
$$

whose components are real-valued phase-space functions

$$
W_{j}=W_{j}(\mathbf{q}, \mathbf{p}), P_{j}=P_{j}(\mathbf{q}, \mathbf{p}), j=1,2
$$

A rather general Hamiltonian function in two dimensions can now be written, in terms of $\omega$, as a Clifford product

$$
H=\frac{1}{2} \omega *_{C} \omega=\frac{1}{2}\left(P_{1}^{2}+P_{2}^{2}\right)+\frac{1}{2}\left(W_{1}^{2}+W_{2}^{2}\right) .
$$


We next introduce the 1-forms

$$
\begin{array}{ll}
f=\frac{1}{\sqrt{2}}\left(e^{1}+i e^{3}\right), & \check{f}=\frac{1}{\sqrt{2}}\left(e^{1}-i e^{3}\right), \\
g=\frac{1}{\sqrt{2}}\left(e^{2}+i e^{4}\right), & \check{g}=\frac{1}{\sqrt{2}}\left(e^{2}-i e^{4}\right) .
\end{array}
$$

These are all nilpotent of order 2

$$
f *_{C} f=0=g *_{C} g, \quad \check{f} *_{C} \check{f}=0=\check{g} *_{C} \check{g}
$$

and satisfy

$$
\begin{aligned}
& \{f, \check{f}\}_{C}=2=\{g, \check{g}\}_{C}, \\
& \{g, \check{f}\}_{C}=0=\{\check{g}, f\}_{C}, \\
& \{g, f\}_{C}=0=\{\check{g}, \check{f}\}_{C},
\end{aligned}
$$

where $\{,\}_{C}$ denotes the Clifford anti-commutator. The relations (6) and (7) imply that the set $\left\{2^{-1 / 2} f, 2^{-1 / 2} g, 2^{-1 / 2} \check{f}, 2^{-1 / 2} \check{g}\right\}$ constitutes a Witt basis of the complexified cotangent space. The first two and the last two elements of this basis span two isotropic subspaces (where $g^{\mathbb{C}}$ induces the zero bilinear map) whose direct sum is the whole cotangent space. Then in terms of the complex-valued functions

$$
C_{1}=\frac{1}{\sqrt{2}}\left(W_{1}+i P_{1}\right), \quad C_{2}=\frac{1}{\sqrt{2}}\left(W_{2}+i P_{2}\right)
$$

and their complex conjugates $\bar{C}_{1}, \bar{C}_{2}$, we define

$$
q_{-}=\bar{C}_{1} f+\bar{C}_{2} g, \quad q_{+}=C_{1} \check{f}+C_{2} \check{g},
$$

such that (4) can be rewritten as $\omega=q_{+}+q_{-}$. By using (6) and (7), one can easily verify that $q_{ \pm}$are also nilpotent, and together with $H$, they close in a simple supersymmetric algebra structure

$$
\begin{aligned}
q_{ \pm} *_{C} q_{ \pm} & =0 \\
H & =\frac{1}{2}\left\{q_{-}, q_{+}\right\}_{C}, \\
{\left[q_{ \pm}, H\right]_{C} } & =0 .
\end{aligned}
$$

The Clifford algebra structure of the exterior bundle of $\mathcal{M}$ enabled us to see the supersymmetry (SUSY) structure in the corresponding classical system. Such systems possessing 
the fermionic (anti-commuting elements) degrees of freedom, in addition to the usual bosonic ones, are known as pseudoclassical models (see [6] for an extensive list of references). They serve as the classical limits of quantum systems having both kinds of degrees of freedom. However, the SUSY structure emerging above does not seem to be promising much at this stage since the third relation of (10) is trivially satisfied. Indeed, $H$ is a 0 -form and therefore Clifford commutes with all forms. In general, the third relation of (10) must be a consequence of the nilpotent nature of so called supercharges $q_{ \pm}$. In what follows, the algebra will be deformed in such a way that the last three relations with a new Hamiltonian, which turns out to be an inhomogeneous even Clifford form comprising a 0-form and a 2-form, will manifest a genuine SUSY structure. For this purpose, the basics of Moyal star-product and its corresponding bracket are briefly reviewed in the following subsection.

\section{Star Product and Moyal Bracket}

In the canonical $(\mathbf{q}, \mathbf{p})$ coordinates, the Moyal $*$-product on $\mathcal{F}$ is defined by

$$
*=\exp \left[\frac{1}{2} i \hbar \sum_{j=1}^{2}\left(\overleftarrow{\partial}_{q_{j}} \vec{\partial}_{p_{j}}-\overleftarrow{\partial}_{p_{j}} \vec{\partial}_{q_{j}}\right)\right]
$$

where $\hbar$ is the Planck constant and $\overleftarrow{\partial}, \vec{\partial}$ are acting, respectively, on the left and on the right. This product is bilinear, associative, and obeys the relation

$$
\left(\overline{F_{1} * F_{2}}\right)=\bar{F}_{2} * \bar{F}_{1}
$$

under complex conjugation. In terms of the $*$-product the Moyal bracket $[,]_{M}$ is defined as

$$
[F, G]_{M}=F * G-G * F
$$

for all phase-space functions. Note that in view of (12), we have

$$
\overline{[F, G]}_{M}=-[\bar{F}, \bar{G}]_{M}
$$

In particular, the Moyal bracket of two real-valued functions is a purely imaginary-valued function. The most important properties of the $*$-product and the MB are the following limiting relations

$$
\begin{aligned}
\lim _{\hbar \rightarrow 0} F * G & =F G, \\
\lim _{\hbar \rightarrow 0} \frac{1}{i \hbar}[F, G]_{M} & =[F, G]_{P},
\end{aligned}
$$


which hold for generic $\hbar$-independent phase-space functions. These reveal the fact that the associative $*$-algebra and the Lie algebra structure of $\mathcal{F}$ given by the MB are, respectively,

deformations (in the sense of Gerstanhaber [18]) of associative algebra structure of $\mathcal{F}$ with respect to pointwise product and of Lie algebra structure determined with respect to PB. All quantum effects are encoded in the $*$-product with respect to which the real elements of $\mathcal{F}$ are promoted to the status of quantum observables.

\section{MOYAL-CLIFFORD ALGEBRA}

So far the components of Clifford forms were commuting quantities since they were multiplied by the ordinary pointwise product. However, one can go over to the non-commutative or quantum case by demanding that the coefficients are to be multiplied by the Moyal *product. This can be achieved by combining (2) and (11) together. The resulting associative product and algebra will be referred to as the Moyal-Clifford (MC) product, denoted by $*_{M C}$ and as the MC-algebra, respectively. We will directly apply this product to the above formulation. In doing so, we would like the first relation of (10) to remain intact with respect to this new product as well.

\section{A. SUSY structure by deformation}

It is easy to show that

$$
\begin{aligned}
& q_{+} *_{M C} q_{+}=\left[C_{1}, C_{2}\right]_{M} \check{f} *_{C} \check{g} \\
& q_{-} *_{M C} q_{-}=\left[\bar{C}_{1}, \bar{C}_{2}\right]_{M} f *_{C} g=-{\overline{\left[C_{1}, C_{2}\right]_{M}}}_{M} *_{C} g
\end{aligned}
$$

where in the last equality we have used (14). Thus $q_{+}$and $q_{-}$are nilpotent with respect to the MC-product if and only if

$$
\left[C_{1}, C_{2}\right]_{M}=0
$$

In terms of $W_{j}$ 's and $P_{j}$ 's, this condition amounts to

$$
\left[W_{1}, W_{2}\right]_{M}-\left[P_{1}, P_{2}\right]_{M}=i\left[W_{2}, P_{1}\right]_{M}-i\left[W_{1}, P_{2}\right]_{M}
$$

Since $W_{j}$ 's and $P_{j}$ 's are real-valued, the left hand side of (16) is, in view of (14), purely imaginary, while the right hand side is real. So the condition (16) is, in fact, equivalent to 
the following two conditions:

$$
\begin{aligned}
{\left[W_{1}, W_{2}\right]_{M} } & =\left[P_{1}, P_{2}\right]_{M}, \\
{\left[W_{1}, P_{2}\right]_{M} } & =\left[W_{2}, P_{1}\right]_{M} .
\end{aligned}
$$

We now define the SUSY Hamiltonian as

$$
H_{s}=\frac{1}{2}\left\{q_{+}, q_{-}\right\}_{M C}
$$

which implies that

$$
\left[q_{ \pm}, H_{s}\right]_{M C}=0
$$

In terms of $\omega_{1}=\omega$ and $\omega_{2}=-i\left(q_{+}-q_{-}\right)$which obey $\left\{\omega_{1}, \omega_{2}\right\}_{M C}=0, H_{s}$ can be factorized as

$$
H_{s}=\frac{1}{2} \omega_{1} *_{M C} \omega_{1}=\frac{1}{2} \omega_{2} *_{M C} \omega_{2}
$$

As will be shown in the following subsection, in an appropriate matrix representation of the Clifford algebra, $\omega_{j}$ are Hermitian supercharges and the symmetry determined by the above super-algebra is 2-extended supersymmetry denoted also by $N=2$ SUSY (where $N$ stands for the number of Hermitian supercharges).

Using (9), (19), conditions (17), (18) and

$$
\begin{aligned}
{\left[\bar{C}_{1}, C_{1}\right]_{M} } & =i\left[W_{1}, P_{1}\right]_{M}, \quad\left[\bar{C}_{2}, C_{2}\right]_{M}=i\left[W_{2}, P_{2}\right]_{M} \\
{\left[\bar{C}_{1}, C_{2}\right]_{M} } & =\left[W_{1}, W_{2}\right]_{M}-i\left[W_{2}, P_{1}\right]_{M}, \\
{\left[\bar{C}_{2}, C_{1}\right]_{M} } & =-\left[W_{1}, W_{2}\right]_{M}-i\left[W_{2}, P_{1}\right]_{M},
\end{aligned}
$$

$H_{s}$ can explicitly be evaluated as

$$
\begin{aligned}
H_{s}= & H_{*}+\frac{1}{2}\left\{\left[W_{1}, P_{1}\right]_{M} e^{13}+\left[W_{2}, P_{2}\right]_{M} e^{24}\right. \\
& \left.+\left[W_{2}, P_{1}\right]_{M}\left(e^{14}+e^{23}\right)+\left[W_{1}, W_{2}\right]_{M}\left(e^{12}+e^{34}\right)\right\},
\end{aligned}
$$

where $H_{*}$ should be read as $H_{*} 1$ with 1 being the unit element of the Clifford algebra and

$$
\begin{aligned}
2 H_{*} & =\left\{\bar{C}_{1}, C_{1}\right\}_{M}+\left\{\bar{C}_{2}, C_{2}\right\}_{M} \\
& =P_{1} * P_{1}+P_{2} * P_{2}+W_{1} * W_{1}+W_{2} * W_{2} .
\end{aligned}
$$


Here $\{,\}_{M}$ stands for the anti-Moyal bracket and we have adopted the abbreviation $e^{j k}=$ $e^{j} *_{C} e^{k}$ which for the product of different orthonormal basis elements becomes $e^{12}=e^{1} \wedge e^{2}$, etc. Note that $H_{s}$ is an inhomogeneous even Clifford form whose bosonic part $H_{*}$ is a zero form and the remaining fermionic part is a 2 -form. The existence of all possible 2 -form basis elements in the fermionic part is a reflection of its generality.

\section{B. Matrix realization}

In terms of the $2 \times 2$ Pauli matrices

$$
\sigma_{1}=\left(\begin{array}{cc}
0 & 1 \\
1 & 0
\end{array}\right), \quad \sigma_{2}=\left(\begin{array}{cc}
0 & -i \\
i & 0
\end{array}\right), \quad \sigma_{3}=\left(\begin{array}{cc}
1 & 0 \\
0 & -1
\end{array}\right),
$$

and of the $2 \times 2$ unit matrix $\mathbf{1}$, we shall use

$$
\begin{aligned}
e^{1}=\left(\begin{array}{cc}
0 & i \sigma_{1} \\
-i \sigma_{1} & 0
\end{array}\right), & e^{2}=\left(\begin{array}{cc}
0 & i \sigma_{3} \\
-i \sigma_{3} & 0
\end{array}\right), \\
e^{3}=\left(\begin{array}{cc}
0 & i \sigma_{2} \\
-i \sigma_{2} & 0
\end{array}\right), & e^{4}=\left(\begin{array}{ll}
0 & 1 \\
1 & 0
\end{array}\right),
\end{aligned}
$$

for the complex Clifford basis defined by (3). This is a representation such that all basis matrices are Hermitian, while $e^{3}$ and $e^{4}$ are symmetric and $e^{1}$ and $e^{2}$ are antisymmetric. If the representations (24) are used in (22), we obtain

$$
H_{s}=\left(\begin{array}{cc}
H_{1} & 0 \\
0 & H_{2}
\end{array}\right)=H_{1} \pi_{+}+H_{2} \pi_{-}
$$

where $\pi_{+}=\operatorname{diag}(\mathbf{1}, 0)$ and $\pi_{-}=\operatorname{diag}(0, \mathbf{1})$ denotes the non-primitive projections and

$$
H_{1}=H_{*} \mathbf{1}+H_{1 F}, \quad H_{2}=H_{*} \mathbf{1}+H_{2 F} .
$$

$H_{j F}$ 's represent the following fermionic parts

$$
\begin{aligned}
H_{1 F} & =\frac{i}{2} B_{+} \sigma_{3} \\
H_{2 F} & =\frac{i}{2} B_{-} \sigma_{3}-i\left[W_{2}, P_{1}\right]_{M} \sigma_{1}-i\left[W_{1}, W_{2}\right]_{M} \sigma_{2}
\end{aligned}
$$


with

$$
B_{ \pm}=\left[W_{1}, P_{1}\right]_{M} \pm\left[W_{2}, P_{2}\right]_{M}
$$

In deriving these relations we made use of $\sigma_{1} \sigma_{2} \sigma_{3}=i \mathbf{1}$ and its consequences. Note that $H_{1}$ is diagonal.

Having obtained matrix realizations of the Hamiltonians we should emphasize that both of the $H_{1}$ and $H_{2}$ are Hermitian. Firstly, in view of (14) $H_{*}$ given by (23) is a real-valued phasespace function. Secondly, as $B_{ \pm}$and the other two coefficient functions of $H_{2 F}$ consisting Moyal brackets are purely imaginary valued, the presence of the imaginary unit $i$ in $(27)$ and (28) ensures the Hermiticity of the both fermionic parts.

Equations (15) (or equivalently (17) and (18)), (20) and (22) show the main differences between the Clifford algebra and the Moyal-Clifford algebra. These restrictions given by (15) arise in order to make $q_{ \pm}$nilpotent in the deformed case and play important roles in the rest of this work. The resulting algebra is a genuine SUSY algebra and the resulting Hamiltonians have non-classical parts. The common bosonic part $H_{*}$ of $H_{1}$ and $H_{2}$ has $H$ given by $(5)$ as its classical limit: $\lim _{\hbar \rightarrow 0} H_{*}=H$. However, their fermionic parts are different and have no classical limits despite the fact that the coefficient functions of the Pauli matrices have classical limits: all Moyal brackets in (27) and (28) reduce to PB brackets in the classical limit after dividing by $i \hbar$.

\section{INTERTWINING, ISO-SPECTRAL PROPERTY AND CONSTANTS OF MO- TION}

In terms of

$$
\begin{aligned}
& L_{1}=C_{1}\left(i \sigma_{1}+\sigma_{2}\right)-i C_{2}\left(\mathbf{1}-\sigma_{3}\right)=2 i\left(\begin{array}{cc}
0 & 0 \\
C_{1} & -C_{2}
\end{array}\right), \\
& L_{2}=C_{1}\left(i \sigma_{1}+\sigma_{2}\right)+i C_{2}\left(\mathbf{1}+\sigma_{3}\right)=2 i\left(\begin{array}{cc}
C_{2} & 0 \\
C_{1} & 0
\end{array}\right),
\end{aligned}
$$

the matrix representations of the supercharges $q_{ \pm}$are found, from (9) and (24), to be

$$
q_{+}=\frac{1}{\sqrt{2}}\left(\begin{array}{cc}
0 & L_{1} \\
-L_{2} & 0
\end{array}\right)={q_{-}{ }^{\dagger} .}^{\dagger} .
$$


The matrices of $\omega_{1}$ and $\omega_{2}$ are Hermitian. Nilpotency of $q_{+}$implies that

$$
L_{1} *_{M C} L_{2}=0=L_{2} *_{M C} L_{1},
$$

i.e. $L_{1}$ and $L_{2}$ are divisors of zero with respect to the $*_{M C}$-product, which denotes, from now on, the star product of matrix-valued phase-space functions. We should note that the second equality of (32) results from the matrix product of factors $i \sigma_{1}+\sigma_{2}$ and $\left(\mathbf{1} \pm \sigma_{3}\right)$ appearing in (29) and (30), while the condition (15) is essential for the first equality of (32).

On the other hand, (20) implies the following double intertwining relations:

$$
\begin{aligned}
& L_{2} *_{M C} H_{1}=H_{2} *_{M C} L_{2}, \\
& L_{1} *_{M C} H_{2}=H_{1} *_{M C} L_{1} .
\end{aligned}
$$

One can easily verify that $\left(D_{1} *_{M C} D_{2}\right)^{\dagger}=D_{2}^{\dagger} *_{M C} D_{1}^{\dagger}$ holds for arbitrary matrix valued functions $D_{j}$ 's. In view of this fact, the Hermitian conjugates of (33) and (34), or equivalently the relation $\left[q_{-}, H_{s}\right]_{M C}=0$, gives the following additional intertwining relations

$$
\begin{aligned}
& L_{2}^{\dagger} *_{M C} H_{2}=H_{1} *_{M C} L_{2}^{\dagger}, \\
& L_{1}^{\dagger} *_{M C} H_{1}=H_{2} *_{M C} L_{1}^{\dagger} .
\end{aligned}
$$

As is evident from (32), $L_{1}^{\dagger}$ and $L_{2}^{\dagger}$ are also divisors of zero. By virtue of (19) and (31), we also obtain

$$
\begin{aligned}
& H_{1}=\frac{1}{4}\left(L_{1} *_{M C} L_{1}^{\dagger}+L_{2}^{\dagger} *_{M C} L_{2}\right), \\
& H_{2}=\frac{1}{4}\left(L_{1}^{\dagger} *_{M C} L_{1}+L_{2} *_{M C} L_{2}^{\dagger}\right),
\end{aligned}
$$

which show that each partner Hamiltonian can be written as a sum of factorized terms.

Let us call a nonzero $2 \times 1$ matrix-valued function $\Psi$ a star-eigenspinor of a $2 \times 2$ matrixvalued function $T$ corresponding to the star-eigenvalue $\lambda$ if and only if $T *_{M C} \Psi=\lambda \Psi$. This definition implies that $\Psi^{\dagger} *_{M C} T^{\dagger}=\bar{\lambda} \Psi^{\dagger}$, and that $\Psi$ is nonzero if and only if $\Psi^{\dagger} *_{M C} \Psi \neq 0$. Then the standard theorems for Hermitian operators, in particular the theorems concerning the reality of spectra and the orthogonality of eigenfunctions corresponding to different eigenvalues, are also valid in the present context. We use the term spinor in the usual sense of $2 \times 1$ matrix eigenfunction and remark that this term has a wider meaning in the nomenclature of Clifford algebra. Bearing these facts in mind, we now return to the physical implications of the intertwining relations [7]. 
The first important implication is the fact that $H_{1}$ and $H_{2}$ are iso-spectral, that is, they have almost the same spectra. More concretely, if $\Psi$ is an eigen-spinor of $H_{1}$ with eigenvalue $\lambda$, then $L_{2} *_{M C} \Psi$ and $L_{1}^{\dagger} *_{M C} \Psi$ are also eigen-spinors of $H_{2}$ with the same eigenvalue provided that $\Psi$ is not in the $M C$-kernel of $L_{2}$, or $L_{1}^{\dagger}$. In view of (34) and (35) the analogous remark is valid for the eigen-spinors of $H_{2}$. For brevity, we refer to the following section for more details and return to another implication of the intertwining relations.

Multiplying one of the relations (33-36) by $L_{j}$ (or $L_{j}^{\dagger}$ ) from the left and comparing the resulting expression with a similarly obtained one from the others, lead to the following matrix valued functions:

$$
\begin{array}{ll}
R_{1}=L_{1} *_{M C} L_{1}^{\dagger}, & S_{2}=L_{2}^{\dagger} *_{M C} L_{2}, \\
R_{2}=L_{2} *_{M C} L_{2}^{\dagger}, & S_{1}=L_{1}^{\dagger} *_{M C} L_{1} .
\end{array}
$$

These commute with $H_{1}$ and $H_{2}$ in the following way:

$$
\begin{aligned}
& {\left[R_{1}, H_{1}\right]_{M C}=0=\left[S_{2}, H_{1}\right]_{M C},} \\
& {\left[R_{2}, H_{2}\right]_{M C}=0=\left[S_{1}, H_{2}\right]_{M C} .}
\end{aligned}
$$

However they are not independent since $4 H_{1}=R_{1}+S_{2}$ and $4 H_{2}=R_{2}+S_{1}$. That is, if there is no explicit time dependence, each system has, together with the Hamiltonian, two constants of motion. The explicit forms of the constants of motion for $H_{1}$ are $S_{2}=2 H_{1}\left(\mathbf{1}+\sigma_{3}\right)$ and $R_{1}=2 H_{1}\left(\mathbf{1}-\sigma_{3}\right)$. Since $H_{1}$ is diagonal, $S_{2}$ and $R_{1}$ are its projected forms.

The method developed so far contains four real-valued phase-space functions. Apart from conditions (17) and (18), there is no constraint on these functions. Therefore, the $*$ products of functions appearing in our formulae contain, in general, countably infinite terms each characterized by a positive power of $\hbar$. This generality enables us to study quantum properties of many physically relevant systems on a classical phase space. However, as we are about to do in the following section, for physically relevant systems some of these functions should be restricted to finite polynomial functions of the canonical coordinates. In such a case a comparison with the existing literature of supersymmetric quantum mechanics, in which majority of application have been carried out in the usual operator formulation of quantum mechanics, can be made. In this regard it is interesting to observe that the forms of our $H_{s}, H_{1}$ and supercharges are similar, up to some permutations of rows and columns of matrices, to that found in [19]. 


\section{APPLICATIONS}

In terms of spin raising and lowering matrices $\sigma_{ \pm}=\left(\sigma_{1} \pm i \sigma_{2}\right) / 2, H_{2 F}$ given by (28) can be rewritten as

$$
H_{2 F}=\frac{i}{2} B_{-} \sigma_{3}+\sqrt{2}\left(\mathcal{A} \sigma_{+}+\overline{\mathcal{A}} \sigma_{-}\right)
$$

where

$$
\mathcal{A}=\left[W_{2}, \bar{C}_{1}\right]_{M}, \quad \overline{\mathcal{A}}=-\left[W_{2}, C_{1}\right]_{M}
$$

These are the phase-space analogues of the bosonic raising and lowering operators when $[\mathcal{A}, \overline{\mathcal{A}}]_{M}$ is a (real) constant. To give illustrative examples we shall (from here on) restrict the study to such cases, take $\hbar=1$ and denote the second term of (43) as

$$
H_{J C}(\mathcal{A})=\sqrt{2}\left(\mathcal{A} \sigma_{+}+\overline{\mathcal{A}} \sigma_{-}\right)
$$

when $[\mathcal{A}, \overline{\mathcal{A}}]_{M}=1$. In such a case, $H_{J C}$ is the phase space analog of the well-known, fully quantum mechanical Jaynes-Cummings (JC) Hamiltonian of quantum optics which describes

a bipartite (a two-level atom and a field) system. In the parlance of quantum optics, $\sigma_{ \pm}$are known as the atomic transition operators and $\sigma_{3}$ as the inversion operator. The first $\mathcal{A} \sigma_{+}$ term at the right-hand side of (45) represents an absorption process in which destruction of a field quanta and a transition to a higher atomic level take place. The second term $\overline{\mathcal{A}} \sigma_{-}$describes an emission process in which creation of a field quanta is accompanied by a transition to a lower atomic level.

Let us see how the phase-space version of such a model together with its supersymmetric partner naturally emerges from the method developed above.

\section{A. Example 1: Jaynes-Cummings-type systems}

A particular simple choice of $W_{j}$ and $P_{1}$ amenable to various physical applications seems to be as

$$
W_{1}=p_{2}, \quad P_{1}=q_{2}, \quad W_{2}=q_{1} p_{2}-q_{2} p_{1}
$$

Then, in view of $\left[W_{1}, W_{2}\right]_{M}=i p_{1}$ and $\left[W_{2}, P_{1}\right]_{M}=-i q_{1}$, the conditions (17) and (18) imply that the most general form of $P_{2}$ is

$$
P_{2}=p_{1} p_{2}+q_{1} q_{2}+K_{1}
$$


such that $K_{1}=K_{1}\left(q_{1}, p_{1}\right)$. It is now straightforward to verify, with $C_{1}=\left(p_{2}+i q_{2}\right) / \sqrt{2}$ from (44), that

$$
\mathcal{A}=-\frac{1}{\sqrt{2}}\left(q_{1}+i p_{1}\right), \quad[\mathcal{A}, \overline{\mathcal{A}}]_{M}=1
$$

Hence, $\mathcal{A}$ and $\overline{\mathcal{A}}$ are bosonic lowering (annihilation) and raising (creation) phase-space functions, respectively. One can also define another such bosonic pair by

$$
\mathcal{B}=-\frac{1}{\sqrt{2}}\left(q_{2}+i p_{2}\right), \quad[\mathcal{B}, \overline{\mathcal{B}}]_{M}=1
$$

which Moyal commute with the above pair. $W_{2}$ represents the angular momentum perpendicular to the $q_{1} q_{2}$-plane and, together with $P_{2}$, can be rewritten as

$$
W_{2}=-i(\overline{\mathcal{A}} \mathcal{B}-\mathcal{A} \overline{\mathcal{B}}), \quad P_{2}=\mathcal{A} \overline{\mathcal{B}}+\overline{\mathcal{A}} \mathcal{B}+K_{1}
$$

Let us define the phase-space number functions

$$
N_{\mathcal{A}}=\overline{\mathcal{A}} * \mathcal{A}=\overline{\mathcal{A}} \mathcal{A}-\frac{1}{2}, \quad N_{\mathcal{B}}=\overline{\mathcal{B}} * \mathcal{B}=\overline{\mathcal{B}} \mathcal{B}-\frac{1}{2}
$$

By making use of

$$
F_{1}(\mathbf{q}) * F_{2}(\mathbf{q})=F_{1}(\mathbf{q}) F_{2}(\mathbf{q}), \quad G_{1}(\mathbf{p}) * G_{2}(\mathbf{p})=G_{1}(\mathbf{p}) G_{2}(\mathbf{p})
$$

and $G *_{M} G=G^{2}$ for $G=\mathbf{a} \cdot \mathbf{p}+F_{1}(\mathbf{q})$ (a is a constant vector), we also find

$$
\begin{aligned}
H_{2 F} & =\frac{i}{2} B_{-} \sigma_{3}+H_{J C}(\mathcal{A}) \\
B_{ \pm} & =i\left[-1 \pm 2\left(N_{\mathcal{B}}-N_{\mathcal{A}}+X_{1}\right)\right] \\
H_{*} & =H_{\mathcal{A}}+2 N_{\mathcal{B}}\left(N_{\mathcal{A}}+1\right)+Y_{1}
\end{aligned}
$$

where $H_{\mathcal{A}}=\left(2 N_{\mathcal{A}}+1\right) / 2$, and

$$
\begin{aligned}
X_{1} & =-\frac{i}{2}\left[W_{2}, K_{1}\right]_{M} \\
Y_{1} & =\frac{1}{2}\left\{\mathcal{A} \overline{\mathcal{B}}+\overline{\mathcal{A}} \mathcal{B}, K_{1}\right\}_{M}+\frac{1}{2} K_{1} * K_{1} .
\end{aligned}
$$

$H_{2}$ is of the type of a JC Hamiltonian [9, 10, 11] describing a two-level atom interacting with a quantized, two-mode electromagnetic field of which only the $\mathcal{A}$-mode directly interacts with the atom and hence causing transitions between levels. $H_{\mathcal{A}}$ in $H_{*}$ is the energy of the $\mathcal{A}$-mode and the remaining part

$$
H_{\text {int }}=H_{*}-H_{\mathcal{A}}=2 N_{\mathcal{B}}\left(N_{\mathcal{A}}+1\right)+Y_{1}
$$


represents the energy of the other mode as well as the interaction between the modes. The super-partners are

$$
\begin{aligned}
& H_{1}=\left(H_{\text {int }}+H_{\mathcal{A}}\right) \mathbf{1}+\left(H_{\mathcal{A}}-N_{\mathcal{B}}\right) \sigma_{3}, \\
& H_{2}=\left(H_{\text {int }}+H_{\mathcal{A}}\right) \mathbf{1}+\left(H_{\mathcal{B}}-N_{\mathcal{A}}\right) \sigma_{3}+H_{J C}(\mathcal{A}),
\end{aligned}
$$

where $H_{\mathcal{B}}=\left(2 N_{\mathcal{B}}+1\right) / 2$, we have taken $K_{1}=0$ and hence $H_{\text {int }}=2 N_{\mathcal{B}}\left(N_{\mathcal{A}}+1\right)$. In such a case the interactions between the modes are through the number functions. By choosing $K_{1}$ such that $X_{1}$ and $Y_{1}$ are nonzero, one can also model certain types of mode interactions [20]. Even in the simplest case when $K_{1}$ is a nonzero constant, $X_{1}$ is zero but the $Y_{1}$ term, which becomes $K_{1}(\mathcal{A} \overline{\mathcal{B}}+\overline{\mathcal{A}} \mathcal{B})+\left(K_{1}^{2} / 2\right)$, represents a coherent exchange of photons between the modes. In any case $H_{1}$ has no JC-type atom-field interaction term.

\section{B. Eigenvalues, Eigenspinors and Wigner Functions}

$H_{1}$ is diagonal and algebraically depends on the complete set $\left\{N_{\mathcal{A}} \mathbf{1}, N_{\mathcal{B}} \mathbf{1}, \sigma_{3}\right\}$ of three mutually commuting (in the MC sense) matrix-valued phase-space functions. Using the shortened notation $(\mathbf{z} \mid \ldots)=(\mathbf{q}, \mathbf{p} \mid \ldots)$, their common eigenspinors will be denoted by

$$
\left(\mathbf{z} \mid j, n_{\mathcal{A}}, n_{\mathcal{B}}\right)=|j\rangle \otimes\left(\mathbf{z} \mid n_{\mathcal{A}}, n_{\mathcal{B}}\right)
$$

Here $n_{\mathcal{A}}, n_{\mathcal{B}}=0,1,2, \ldots$, stand for the numbers of mode-quanta and $j(=1,2)$ labels the bare states of atom such that $|j\rangle$ 's, like the spin-up and spin-down states of a spin-1/2 system, satisfy $\sigma_{3}|1\rangle=-|1\rangle, \sigma_{3}|2\rangle=|2\rangle$ and

$$
\begin{aligned}
& \sigma_{-}|1\rangle=0=\sigma_{+}|2\rangle, \\
& \sigma_{-}|2\rangle=|1\rangle, \\
& \sigma_{+}|1\rangle=|2\rangle .
\end{aligned}
$$

In (57) the real-valued functions $\left(\mathbf{z} \mid n_{\mathcal{A}}, n_{\mathcal{B}}\right)$ represent the diagonal Wigner functions [21, 22]

$$
\left(\mathbf{z} \mid n_{\mathcal{A}}, n_{\mathcal{B}}\right)=\frac{1}{n_{\mathcal{A}} ! n_{\mathcal{B}} !} \overline{\mathcal{A}}^{n_{\mathcal{A}}} *_{M} \overline{\mathcal{B}}^{n_{\mathcal{B}}} *_{M}(\mathbf{z} \mid 0,0) *_{M} \mathcal{A}^{n_{\mathcal{A}}} *_{M} \mathcal{B}^{n_{\mathcal{B}}}
$$

for two-mode field. Here $(\mathbf{z} \mid 0,0)$ denotes the vacuum Wigner function defined by

$$
\mathcal{A} *_{M}\langle\mathbf{z} \mid 0,0\rangle=0=\mathcal{B} *_{M}\langle\mathbf{z} \mid 0,0\rangle .
$$


The normalized (its integral all over the phase space set to 1) solution of these equations is $\pi^{-2} \exp -\left(p_{1}^{2}+p_{1}^{2}+q_{1}^{2}+q_{1}^{2}\right)$. Explicit functional forms of the higher level Wigner functions are proportional to [23]

$$
L_{n_{\mathcal{A}}}(4 \mathcal{A} \overline{\mathcal{A}}) L_{n_{\mathcal{B}}}(4 \mathcal{B} \overline{\mathcal{B}})\langle\mathbf{z} \mid 0,0\rangle
$$

where $L_{k}$ stands for the Laguerre polynomials. These are products of the harmonic oscillator Wigner functions which are well known [24].

One can also verify

$$
\begin{aligned}
\mathcal{A} *_{M}\left(\mathbf{z} \mid n_{\mathcal{A}}, n_{\mathcal{B}}\right) & =\sqrt{n_{\mathcal{A}}}\left(\mathbf{z} \mid n_{\mathcal{A}}-1, n_{\mathcal{B}}\right) \\
\overline{\mathcal{A}} *_{M}\left(\mathbf{z} \mid n_{\mathcal{A}}, n_{\mathcal{B}}\right) & =\sqrt{n_{\mathcal{A}}+1}\left(\mathbf{z} \mid n_{\mathcal{A}}+1, n_{\mathcal{B}}\right), \\
N_{\mathcal{A}} *_{M}\left(\mathbf{z} \mid n_{\mathcal{A}}, n_{\mathcal{B}}\right) & =n_{\mathcal{A}}\left(\mathbf{z} \mid n_{\mathcal{A}}, n_{\mathcal{B}}\right) .
\end{aligned}
$$

Similar relations hold for $N_{\mathcal{B}}, \mathcal{B}$ and $\overline{\mathcal{B}}$. In view of these relations, the eigenvalues of $H_{1}$

$$
\begin{aligned}
& H_{1} *_{M C}\left(\mathbf{z} \mid 1, n_{\mathcal{A}}, n_{\mathcal{B}}\right)=\lambda_{1 n_{\mathcal{A}} n_{\mathcal{B}}}\left(\mathbf{z} \mid 1, n_{\mathcal{A}}, n_{\mathcal{B}}\right) \\
& H_{1} *_{M C}\left(\mathbf{z} \mid 2, n_{\mathcal{A}}, n_{\mathcal{B}}\right)=\lambda_{2 n_{\mathcal{A}} n_{\mathcal{B}}}\left(\mathbf{z} \mid 1, n_{\mathcal{A}}, n_{\mathcal{B}}\right)
\end{aligned}
$$

are easily found, from (55) and (59), to be

$$
\lambda_{1 n_{\mathcal{A}} n_{\mathcal{B}}}=n_{\mathcal{B}}\left(2 n_{\mathcal{A}}+3\right), \quad \lambda_{2 n_{\mathcal{A}} n_{\mathcal{B}}}=\left(n_{\mathcal{B}}+1\right)\left(2 n_{\mathcal{A}}+1\right)
$$

For $n_{\mathcal{A}}=0=n_{\mathcal{B}}$, we have $\lambda_{100}=0$ and $\lambda_{200}=1$ which represent the bare energy levels of the atom. In this case, $H_{1}$ is simply $\left(\mathbf{1}+\sigma_{3}\right) / 2$. The level $\lambda_{1 n_{\mathcal{A}} 0}=0$ is infinitely degenerate whereas all the other levels are finitely degenerate. Provided that $n_{\mathcal{A}} \geq n_{\mathcal{B}}$, the level $\lambda_{2 n_{\mathcal{A}} n_{\mathcal{B}}}$ is the higher level of the whole atom-field system. These degeneracies arise from the fact that we have taken the frequencies of the modes to be equal (degenerate modes). Evidently, these discussions can be extended to general case in which the frequencies are different.

For this example, we have $C_{2}=i \sqrt{2} \overline{\mathcal{B}} \mathcal{A}, C_{1}=-i \overline{\mathcal{B}}$ and by virtue of

$$
2 \sigma_{+} \sigma_{-}=\mathbf{1}+\sigma_{3}, \quad 2 \sigma_{-} \sigma_{+}=\mathbf{1}-\sigma_{3}
$$

the intertwining (matrix-valued) functions, given by (29) and (30) can be rewritten as

$$
\begin{array}{ll}
L_{1}=2 \overline{\mathcal{B}} \sigma_{-}\left(\mathbf{1}+\sqrt{2} \mathcal{A} \sigma_{+}\right), & L_{1}^{\dagger}=2 \mathcal{B}\left(\mathbf{1}+\sqrt{2} \overline{\mathcal{A}} \sigma_{-}\right) \sigma_{+} \\
L_{2}=2 \overline{\mathcal{B}}\left(\mathbf{1}-\sqrt{2} \mathcal{A} \sigma_{+}\right) \sigma_{-}, & L_{2}^{\dagger}=2 \mathcal{B} \sigma_{+}\left(\mathbf{1}-\sqrt{2} \overline{\mathcal{A}} \sigma_{-}\right)
\end{array}
$$


Now it is easy to verify

$$
L_{2} *_{M C}\left(\mathbf{z} \mid 1, n_{\mathcal{A}}, n_{\mathcal{B}}\right)=0=L_{1}^{\dagger} *_{M C}\left(\mathbf{z} \mid 2, n_{\mathcal{A}}, n_{\mathcal{B}}\right),
$$

which simply follow from the actions of $\sigma_{ \pm}$on the atomic bare states. However, one needs to be more careful for the verifications of

$$
\begin{aligned}
& L_{1}^{\dagger} *_{M C}\left(\mathbf{z} \mid 1, n_{\mathcal{A}}, n_{\mathcal{B}}\right)=2 \sqrt{n_{\mathcal{B}}} \Phi_{n_{\mathcal{A}} n_{\mathcal{B}}}(\mathbf{z}) \\
& L_{2} *_{M C}\left(\mathbf{z} \mid 2, n_{\mathcal{A}}, n_{\mathcal{B}}\right)=2 \sqrt{n_{\mathcal{B}}+1} \Psi_{n_{\mathcal{A}} n_{\mathcal{B}}}(\mathbf{z})
\end{aligned}
$$

where

$$
\begin{aligned}
& \Phi_{n_{\mathcal{A}} n_{\mathcal{B}}}(\mathbf{z})=\sqrt{2 n_{\mathcal{B}}+2}\left(\mathbf{z} \mid 1, n_{\mathcal{A}}+1, n_{\mathcal{B}}-1\right)+\left(\mathbf{z} \mid 2, n_{\mathcal{A}}, n_{\mathcal{B}}-1\right) \\
& \Psi_{n_{\mathcal{A}} n_{\mathcal{B}}}(\mathbf{z})=\left(\mathbf{z} \mid 1, n_{\mathcal{A}}, n_{\mathcal{B}}+1\right)-\sqrt{2 n_{\mathcal{A}}}\left(\mathbf{z} \mid 2, n_{\mathcal{A}}-1, n_{\mathcal{B}}+1\right) .
\end{aligned}
$$

As a consistency check, one can directly verify that these are the (unnormalized) eigenspinors of $\mathrm{H}_{2}$

$$
\begin{aligned}
& H_{2} *_{M C} \Phi_{n_{\mathcal{A}} n_{\mathcal{B}}}(\mathbf{z})=\lambda_{1 n_{\mathcal{A}} n_{\mathcal{B}}} \Phi_{n_{\mathcal{A}} n_{\mathcal{B}}}(\mathbf{z}) \\
& H_{2} *_{M C} \Psi_{n_{\mathcal{A}} n_{\mathcal{B}}}(\mathbf{z})=\lambda_{2 n_{\mathcal{A}} n_{\mathcal{B}}} \Psi_{n_{\mathcal{A}} n_{\mathcal{B}}}(\mathbf{z})
\end{aligned}
$$

with the same eigenvalues as given by (60). These fully establish the iso-spectral property of $H_{1}$ and $H_{2}$, which was compactly expressed by the SUSY algebra given by (19-21).

In the nomenclature of quantum optics the *-eigenstates given by (64) and (65) are the phase-space analogues of the so-called stationary dressed states or JC-doublet. The states at the right-hand sides of (64) and (65) are known as the bare states of the atom-field system. The latter are the phase-space version of the product states of the bare atom and field states. Finally, we compute, with the help of (62) and (63), the constants of motion

$$
\begin{aligned}
R_{1} & =8 N_{\mathcal{B}}\left(H_{\mathcal{A}}+1\right) \sigma_{-} \sigma_{+} \\
S_{1} & =4\left(N_{\mathcal{B}}+1\right)\left[\sigma_{+} \sigma_{-}+2 N_{\mathcal{B}} \sigma_{-} \sigma_{+}+H_{J C}(\mathcal{A})\right]
\end{aligned}
$$

such that $\left[R_{1}, H_{1}\right]_{M C}=0=\left[S_{1}, H_{2}\right]_{M C}$.

\section{Example 2: Non-resonant JC-type interactions}

For $W_{1}=p_{1}, P_{1}=-q_{1}$ and $W_{2}$ as in (46), the conditions (17) and (18) are satisfied, provided that

$$
P_{2}=p_{1} p_{2}+q_{1} q_{2}+K_{2}
$$


Here $K_{2}=K_{2}\left(q_{2}, p_{2}\right)$ is an arbitrary real function of its arguments. Hence $\sqrt{2} C_{1}=p_{1}-i q_{1}$ and

$$
\left[W_{2}, \bar{C}_{1}\right]_{M}=-\left(q_{2}-i p_{2}\right) / \sqrt{2}=\overline{\mathcal{B}},
$$

where $\mathcal{B}$ and $\overline{\mathcal{B}}$ are defined by (49). As a result, by interchanging the lowering functions of subsection V.A such that $(\mathcal{A}, \mathcal{B}) \leftrightarrow(\overline{\mathcal{B}}, \overline{\mathcal{A}})$, or by direct computations, we find

$$
\begin{aligned}
H_{2 F} & =\frac{i}{2} B_{-} \sigma_{3}+H_{J C}(\overline{\mathcal{B}}), \\
H_{J C}(\overline{\mathcal{B}}) & =\sqrt{2}\left(\overline{\mathcal{B}} \sigma_{+}+\mathcal{B} \sigma_{-}\right), \\
B_{ \pm} & =i\left[1 \pm 2\left(N_{\mathcal{B}}-N_{\mathcal{A}}+X_{2}\right)\right], \\
H_{*} & =H_{\mathcal{B}}+2 N_{\mathcal{A}}\left(N_{\mathcal{B}}+1\right)+Y_{2} .
\end{aligned}
$$

$W_{2}$ and the first term of $P_{2}$ are still given by (50), and $X_{2}, Y_{2}$ are defined as in (53) and (54) provided that $K_{1}$ is replaced by $K_{2}$. For $K_{2}=0$, the Hamiltonians and the corresponding eigenvalues are

$$
\begin{aligned}
H_{1} & =\left(H_{\text {int }}+H_{\mathcal{B}}\right) \mathbf{1}-\left(H_{\mathcal{B}}-N_{\mathcal{A}}\right) \sigma_{3}, \\
H_{2} & =\left(H_{\text {int }}+H_{\mathcal{A}}\right) \mathbf{1}-\left(H_{\mathcal{A}}-N_{\mathcal{B}}\right) \sigma_{3}+H_{J C}(\overline{\mathcal{B}}), \\
\lambda_{1 n_{\mathcal{A}} n_{\mathcal{B}}} & =\left(n_{\mathcal{A}}+1\right)\left(2 n_{\mathcal{B}}+1\right), \lambda_{2 n_{\mathcal{A}} n_{\mathcal{B}}}=n_{\mathcal{A}}\left(2 n_{\mathcal{B}}+1\right) .
\end{aligned}
$$

These are related to $(60)$ by the interchange $\left(1, n_{\mathcal{A}}, n_{\mathcal{B}}\right) \leftrightarrow\left(2, n_{\mathcal{B}}, n_{\mathcal{A}}\right)$.

Now some remarks for the above applications are in order.

The JC-type Hamiltonians originate from the interaction of the electric dipole moment of an atom with a quantized light in dipole approximation and, in general, both $H_{J C}(\mathcal{A})$ and $H_{J C}(\overline{\mathcal{A}})$ take part in such an interaction. To see this we should emphasize that dipole moment has non-vanishing matrix elements only between states of opposite parity. Therefore, the atomic bare states $|1\rangle$ and $|2\rangle$ are assumed of opposite parity and hence the dipole moment is proportional to $\sigma_{+}+\sigma_{-}=|1\rangle\langle 2|+| 2\rangle\langle 1|$. On the other hand since a quantized single mode cavity field is a multiple of $\mathcal{A}+\overline{\mathcal{A}}$ then the dipole energy is proportional to

$$
\left(\sigma_{+}+\sigma_{-}\right)(\mathcal{A}+\overline{\mathcal{A}})=H_{J C}(\mathcal{A})+H_{J C}(\overline{\mathcal{A}})
$$

However, the resonant processes described by $H_{J C}(\mathcal{A})$ are more efficient than the nonresonant processes described by $H_{J C}(\overline{\mathcal{A}})$, especially in the case of a quantized single-mode light. Neglect of the latter is usually called the rotating wave approximation. On the other 
hand, in the case of multi-mode interactions, both types of terms have important physical implications. Here we will be content with pointing out that the above two examples can be combined and then extended in various ways to study the generalized models of quantum optics, such as $N$-atom JC models [25] (also known as Dick models [26]) and spin-boson systems [27]. In particular, time can be included and the dynamics of transitions between atomic levels for both partner Hamiltonians can be studied in the phase space.

\section{CONCLUDING REMARKS}

The Moyal-Clifford algebra, that is, Clifford algebra endowed with the star product of deformation quantization, provides a unified framework to realize the SUSY techniques in a classical phase space, to see relations of various models described by matrix Hamiltonians and to study their phase-space characteristics in great detail. To emphasize some merits of such an approach we recall that in the usual operator formulation of quantum mechanics, Wigner functions are indirectly obtained from wave functions through some convolution integrals which, apart from simple few cases, are not easy to cope with. But, in the deformation quantization they are directly obtained from star-eigenvalue equations. Moreover, by realizing the SUSY techniques in this framework Wigner functions of some delicate states such as the dressed states can be easily obtained by the intertwining functions.

In the presented examples the phase space is spanned, instead of the usual canonically conjugate coordinates of a particle system, by the conjugate amplitudes of mode functions of a quantized electromagnetic field that are dynamically equivalent to conjugate coordinates of a mechanical oscillator. The form of $H_{1}$ and $H_{2}$ and the presented examples may be convincing enough that a lot of physically relevant (charged or uncharged) particle systems can be identified as spacial cases. As much as exhaustive search in this direction seems to be rather involved and will be deferred to a later publication.

\section{Acknowledgments}

We are grateful to anonymous referees for their useful comments and to Ö. Sarığlu for a critical reading of the manuscript and for illuminating discussions. Special thanks are due to A. U. Yılmazer for useful conversations. This work was supported in part by the Scientific 
and Technological Research Council of Turkey (TÜBİTAK).

[1] Bayen, F., Flato, M., Fronsdal, C., Lichnerowicz, A., Sternheimer, D. 1978, Ann. Phys. (NY), 111, 61 and 111.

[2] Zachos, C., Fairlie, D., Curtright, T. (Eds) 2005, Quantum Mechanics in Phase space: An Overview with Selected Papers, Vol 34 (World Scientific).

[3] Henselder, P., Hirshfeld, A. C., Spernat, T. 2005, Ann. Phys. (NY), 317, 107.

[4] Gendensthĕn, L. É., Krive, I. V., Supersymmetry in quantum mechanics 1985, Sov. Phys. Usp., 28, 645-666.

[5] Cooper, F., Khare, A., Sukhatme, U. 1995, Phys. Rep., 251, 267.

[6] Junker, G. 1996, Supersymmetric Methods in Quantum and Statistical Physics (Springer).

[7] Kuru, Ş., Teğmen, A., Verçin, A. 2001, J. Math. Phys., 42, 3344.

[8] Henselder, P. 2007, Phys. Lett. A , 363, 378.

[9] Jaynes, E. T., Cummings, F. W. 1963, Proc. IEEE, 51, 89.

[10] Shore, B. W. and Knight, P. L. 1993, J. Mod. Opt., 40, 1195.

[11] Schleich, W. P. 2001, Quantum Optics in Phase Space (Wiley-VCH).

[12] Fink, J. M., Göpp, M., Baur, M., Bianchetti, R., Leek, P.J., Blais, A., Wallraff, A. 2008, Nature, 454, 315 .

[13] Benn, I. M., Tucker, R. W. 1987, An Introduction to Spinors and Geometry with Applications in Physics (IOP, Bristol).

[14] Arnold, V. I. 1989, Mathematical Methods of Classical Mechanics (2nd ed.; Springer).

[15] Artin, E. 1957, Geometric Algebra (Interscience Publishers).

[16] Hestenes, D. 1966, Space-Time Algebra (Gordon and Breach).

[17] Hestenes, D. 1993, in Spinor, Twistor, Clifford Algebras and Quantum Deformations, ed. Z. Oziewicz et al. (Kluwer) 203.

[18] M. Gerstenhaber, S. D. Schack 1988, in Deformation Theory of Algebras and Structures and Applications, ed. M. Hazewinkel, M. Gerstanhaber, (Kluwer Academic Publishers).

[19] Ioffe, M. V. 2004, J. Phys. A: Math. Gen. 37, 10363.

[20] Barnett, S. M., Radmore, P. M. 1997, Methods in Theoretical Quantum Optics (ClarendonPress). 
[21] Curtright, T., Fairlie, D., Zachos, C. 1998, Phys. Rev. D, 58, 025002.

[22] Curtright, T., Uematsu, T., Zachos, C. 2001, J. Math. Phys., 42, 2396.

[23] Demircioğlu, B., Verçin, A. 2003, Ann. Phys. (NY), 305, 1.

[24] Bartlett, M. S., Moyal, J. E. 1949, Proc. Camb. Phil. Soc., 45, 545.

[25] Chagas, E. A., Fruya, K. 2008, Phys. Lett. A, 372, 5564.

[26] Lambert, N., Emary, C., Brandes, T. 2004, Phys. Rev. Lett., 92, 073602.

[27] Brandes, T. 2005, Phys. Rep., 408, 315. 